\title{
Two-Stage Alignment of fMRI Time Series Using the Experiment Profile to Discard Activation-Related Bias
}

\author{
L. Freire ${ }^{1,2,3}$ and J.-F. Mangin ${ }^{1}$ \\ 1 Service Hospitalier Frédéric Joliot, CEA, 91401 Orsay, France \\ 2 Instituto de Biofísica e Engenharia Biomédica, FCUL, 1749-016 Lisboa, Portugal \\ 3 Instituto de Medicina Nuclear, FML, 1649-028 Lisboa, Portugal
}

\begin{abstract}
In this paper, we show that the standard point of view of the neuroimaging community about fMRI time series alignment should be revisited to overcome the bias induced by activations. We propose to perform a two-stage alignment. The first motion estimation is used to infer a mask of activated areas. The second motion estimation discards these areas during the similarity measure estimations. Simulated and actual time series are used to show that this dedicated approach is more efficient than standard robust similarity measures.
\end{abstract}

\section{Introduction}

In functional MRI (fMRI) activation studies, motion correction is a required pre-processing step, in order to accurately compensate for subject motion during data acquisition. However, it is known that standard registration is often not sufficient to correct for all signal changes due to subject motion. Serious confounds may appear due to the "spin history" effect, or due to interaction between motion and susceptibility artifacts. We have also recently shown that the presence of activated regions may introduce a systematic bias in the motion correction parameters when using L2-metrics based similarity measures, even in the absence of subject motion [1]. According to its amplitude, this motionindependent artifact, which stems from the fact that activated areas behave like biasing outliers, may create spurious activations after the time series alignment, especially along high contrast edges.

A second study has shown that robust similarity measures could highly reduce the amplitude of this task correlated motion correction artifact [2]. While this amplitude reduction was sufficient to get rid of spurious activations in the studied cases, the estimated motion parameters were still correlated with the cognitive task timing. This bias prevents the use of the motion parameters as regressor of non-interest during the following activation inference. Moreover this observation means that the problem is not fully overcome by the robust similarity measures. This weakness is due to the fact that the signal variations occurring in activated areas are often at the noise level. Hence, the simple mechanisms 
underlying robust similarity measures are not sufficient to discard the influence of the whole activated area.

In this paper, we propose to go one step further to devise an fMRI alignment method completely robust to the presence of activations. This approach is maybe the more straightforward at first glance: discard the voxels included in the activated area detected by a first rapid statistical inference. This approach is more cumbersome than the standard ones because it requires a first realignment and some knowledge about the experiment timing. The computation time remains however reasonable because the first realignment/inference sequence can be performed with very simple methods. The resulting activated area used to modify the similarity measure, indeed, does not need to be perfect.

This approach is illustrated first using a motion-free simulated time series including artificial activation-like signal changes based on a periodical paradigm. The improvement is finally highlighted with three actual time series obtained from a $3 \mathrm{~T}$ magnet. All the experiments are performed using five different standard similarity measures.

\section{Materials and Methods}

\section{1 fMRI Acquisitions}

The three fMRI time series used in the paper were acquired on a Brucker scanner operating at $3 \mathrm{~T}$. Volume geometry consists of 18 contiguous slices (slice array $64 \times 80$ ), with in-plane resolution of $3.75 \mathrm{~mm}$ and slice thickness of $5.00 \mathrm{~mm}$. The experimental paradigm was based on an on/off design consisting of two alternating visual stimuli, with period of 18 frames $(2 \mathrm{~s} /$ frame). The time series include 10 periods plus 12 initial frames used to reach the scanner steady state.

\subsection{Similarity Measures}

In this work, five very usual similarity measures were chosen to evaluate the benefit introduced by the proposed approach. Each similarity measure belongs to a different family of the taxonomy described by Roche [3], namely: intensity conservation (difference of squares denoted by LS [4]), intensity conservation with outliers (Geman-McClure M-estimator denoted by GM) 5]; affine relationship (ratio of image uniformity denoted by RIU) [6]; functional relationship (correlation ratio denoted by CR) [7]; and statistical relationship (mutual information denoted by MI) [89]. All the registration methods share the same computational framework (implemented in C language), which includes a cubic spline based interpolation method [10.11] and a Powell like optimization method. For GM, the cut-off value, $\mathrm{C}$, was set to $0.5 \%$ of mean brain value. The smoothing Gaussian kernels applied to the data before registration to increase robustness have $8 \mathrm{~mm}$ width for LS, and $4 \mathrm{~mm}$ width for GM and RIU. No smoothing was applied for $\mathrm{CR}$ and MI, according to the experiments described in [2]. 


\subsection{Discarding Activated Regions}

The proposed new approach is based on the identification of a mask supposed to include all activated areas. All the voxels of this mask are discarded from the similarity measure's evaluation during the final motion estimation. It should be noted that this mask may include some spurious activations stemming from a biased initial motion correction, which is not a problem. Whatever the time series, the sketch of the performed experiment is the following:

1. Apply the standard motion estimation procedures using each of the 5 standard similarity measures. These estimations are the references to which will be compared the results of the new approach;

2. Use the initial motion estimation given by LS to resample the time series (here we have chosen to perform only one initial resampling according to the estimation given by the fastest but also the less robust registration method; hence the same mask was used in each case; of course, in actual applications, the same robust registration method could be used for the two stages);

3. Perform statistical inference from the resampled time series, in order to obtain a coarse estimation of activated regions (A1 activation pattern); in this paper, SPM99 was used but simpler methods should be sufficient;

4. Dilate the activation pattern A1; for the experiments described in this paper, A1 26-neighborhood was added to the mask. This dilation is used first to reduce the number of false negatives, second to help the registration methods that perform a smoothing before the measure estimation. This smoothing, indeed, corrupts a lot of voxels with the activation profile;

5. Perform a second motion estimation from the initial time series using each of the 5 adapted similarity measures. This adaptation consists of discarding the voxels of the dilated activation pattern;

6. For each registration method, compute the cross-correlation of the 6 estimated motion parameters with the cognitive task profile.

\section{Experiments}

\subsection{Simulated Activations}

The benefit of the proposed approach is firstly evaluated with an artificial time series designed to simulate an activation area in the absence of subject motion. This was done duplicating the reference image of the first actual time-series 40 times and adding an activation-like signal change in order to mimic a cognitive activation process. The activation pattern was obtained from statistical inference of one actual time series related to a visual experiment. In order to simulate the effects of thermal noise, Rician noise obtained with two Gaussian distributions in real and complex axis with SD corresponding to $2 \%$ of mean brain value, was added. The results indicate a clear reduction in correlation values for the activation detection-based method whatever the similarity measure (see Fig. 1 and Table 1). The number of activated voxels discarded from the similarity measure estimation, given as a percentage of the brain, was $14 \%$. 


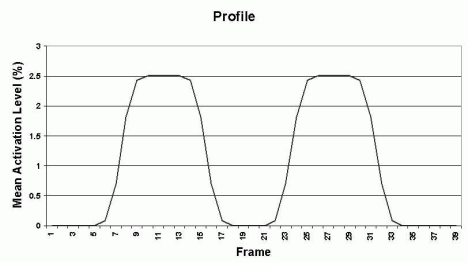

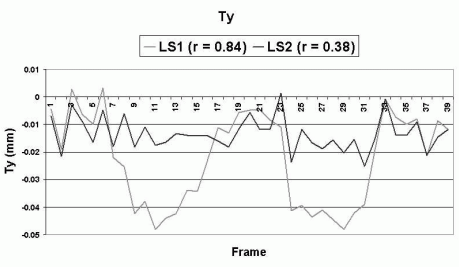

Ty

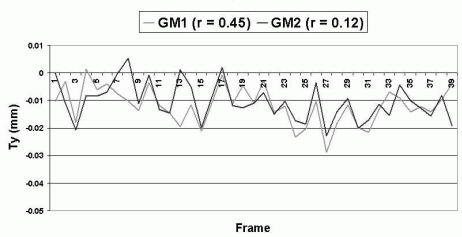

Ty

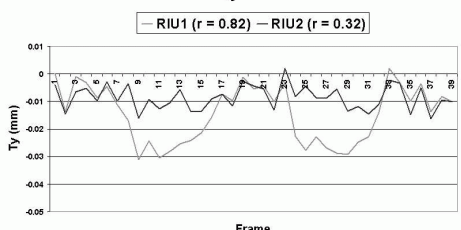

Ty

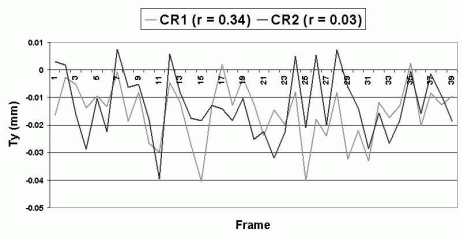

Ty

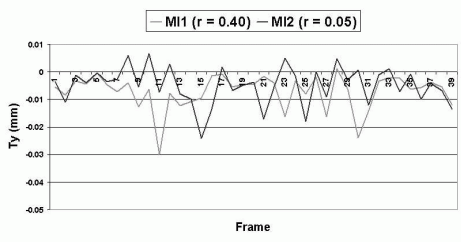

$8 x$

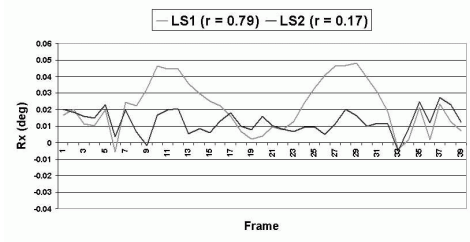

$\mathrm{Rx}$

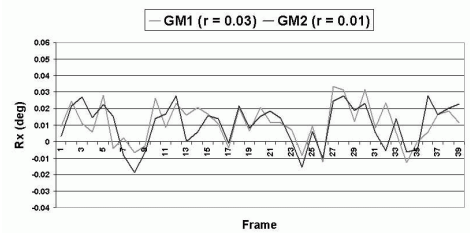

$\mathrm{Rx}$

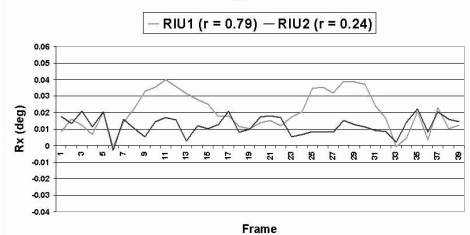

$\mathrm{Rx}$

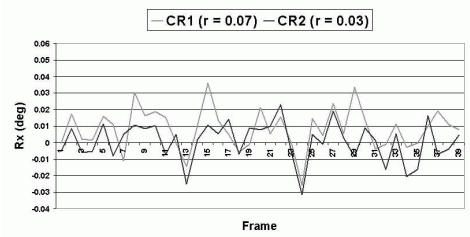

$\mathrm{Rx}$

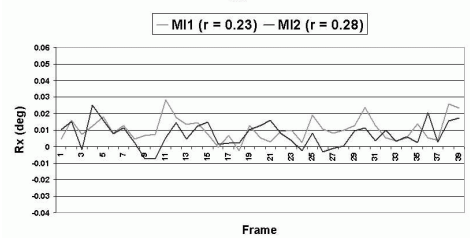

Fig. 1. Registration parameters $t_{y}$ and $r_{x}$ for simulated time series. From top to bottom: activation profile, LS, GM, RIU, CR and MI. For each similarity measure, (1) stands for conventional registration method and (2) for the proposed approach. 
Table 1. Correlation values for the simulated time-series. For each similarity measure, (1) refers to the conventional registration method and (2) for the proposed approach.

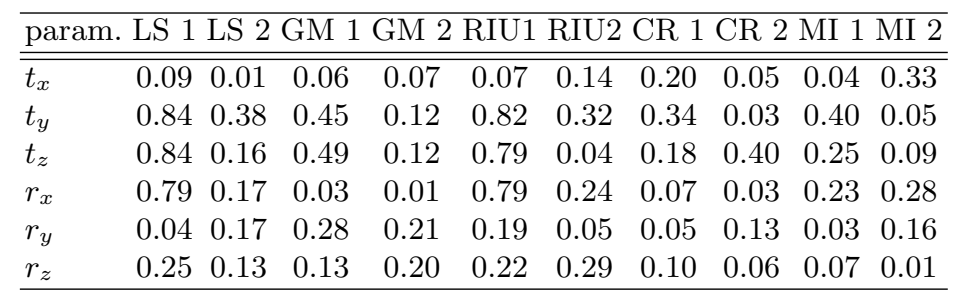

\subsection{Experiments with Actual Time Series}

The same experiment was performed with three different actual time series. For these data, the activation profile used to compute cross-correlation was obtained by the convolution of the task timing with SPM99 hemodynamic model. A moving average was removed from the estimated parameter before computing the correlation in order to discard slow motion trends features. The number of activated voxels in the mask is respectively $19 \%, 22 \%$ and $18 \%$ of the brain size. Fig. 2 presents the results for $t_{y}$ and $r_{x}$ (pitch), for one of the time series. Clear reductions in the correlation with activation paradigm are visible for the different methods, particularly for LS and RIU, the most biased ones. Table 2 summarizes the correlation coefficients for the three data sets.

\section{Discussion}

The work presented in this paper has been triggered by recurrent difficulties observed in our institution relative to alignment by SPM of time series acquired with our 3T magnet [1. The observation of estimated motion parameters perfectly correlated with the cognitive experiment led us to suspect a bias of the registration method. This bias, however, was not systematic. For the experiment used in this paper, a high amplitude bias was clearly observed for only 3 different subjects among 14. Hence, while some simulations had shown that activated areas could father a similar bias, the existence of an actual motion for these 3 subjects was still possible. The results obtained from the experiments performed in this paper definitively rule out this explanation. Discarding indeed about $20 \%$ of the voxels almost remove the correlation with the task.

Since most of the current fMRI realignments are performed using LS based method (SPM and AIR), our result is rather alarming. Fortunately, the bias is highly related to the field strength, and we did not observe such problem with our 1.5T magnet. Our results, however, call for a refinement of the current packages, which should not be difficult. A few parameters have also to be tuned to make our approach more robust. For instance, the dilation of the activated area seems too ad hoc and should be replaced by an activation detection taking into account the smoothing performed during motion estimation. The mask dilation has an 


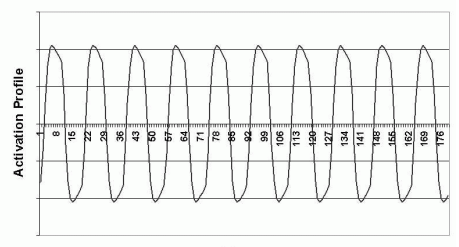

Frame
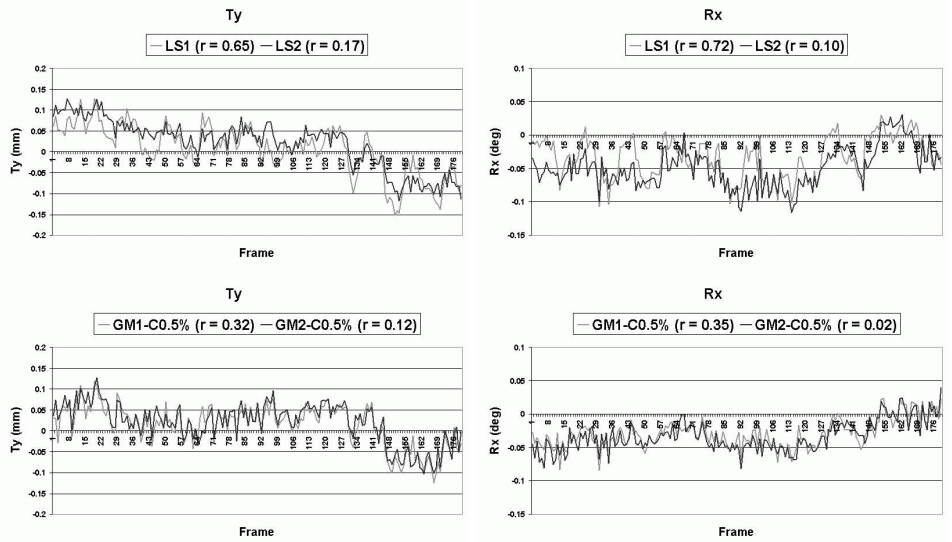

Ty

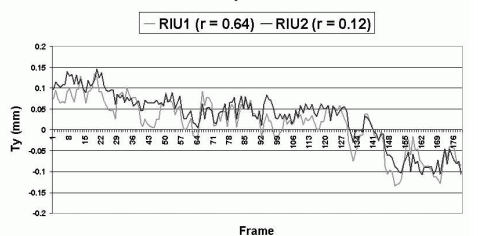

Rx

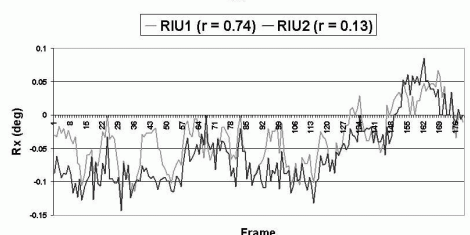

Ty

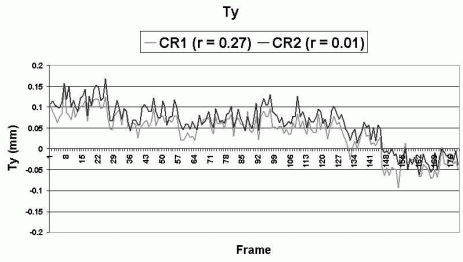

Ty
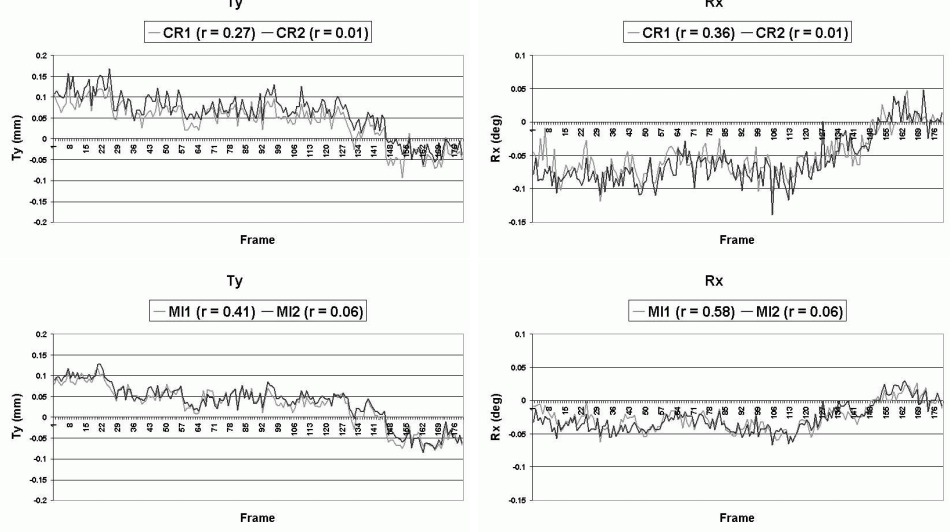

$\mathrm{Rx}$

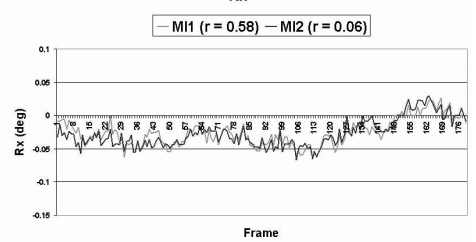

Fig. 2. Registration parameters $t_{y}$ and $r_{x}$ for the first actual time series. From top to bottom: activation profile, LS, GM, RIU, CR and MI. For each similarity measure, (1) stands for conventional registration method and (2) for the proposed approach. 
Table 2. Correlation values for the three actual time-series. For each similarity measure, (1) stands for conventional registration method and (2) for the proposed approach.

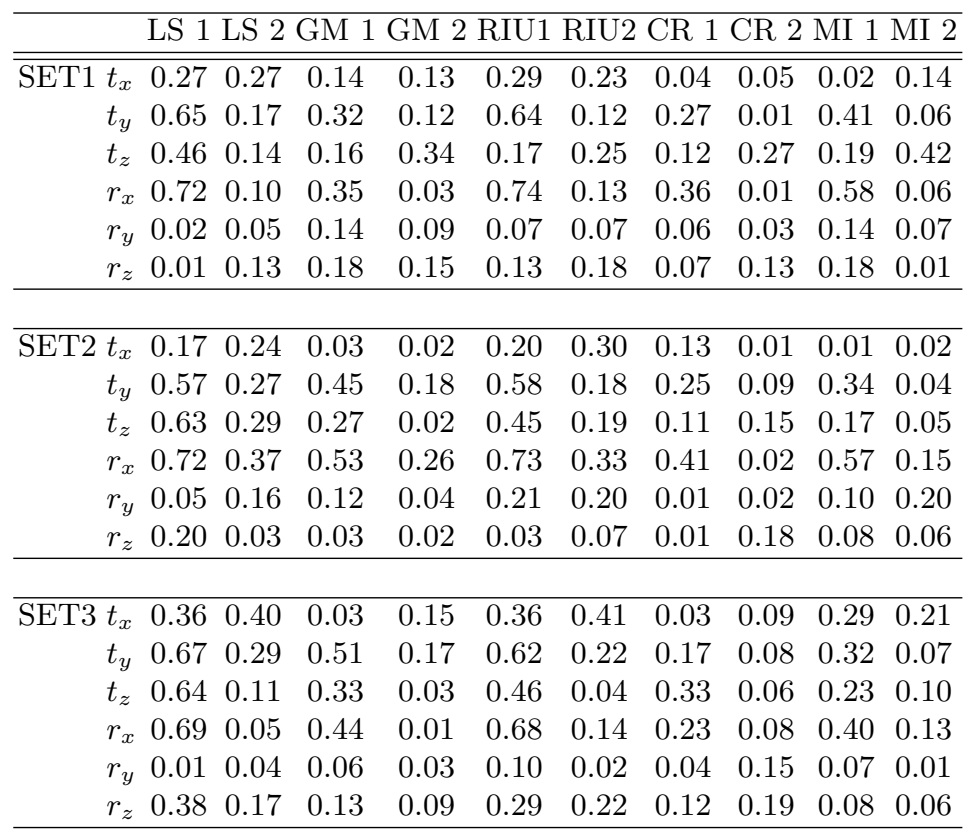

important positive effect. Using a non-dilated mask, for instance, the correlation coefficients for LS and RIU are much higher $(0.26,0.52,0.07,0.41,0.09$ and 0.20$)$ and $(0.26,0.52,0.10,0.49,0.06$ and 0.27$)$, respectively, for the first data set. In the standard registration packages, in fact, a large smoothing is applied to reduce the occurrence of local minima during the estimation of the motion parameter. It has been shown, however, that this smoothing was largely increasing the bias amplitude 12. A careful implementation of the method presented in this paper should allow to discard these smoothing related problems.

Another important point is related to the issue of motion estimation variability. Increasing the number of voxels to be discarded is bound to decrease the method accuracy, because the remaining voxels have a non-uniform localization in the brain. The rigid body hypothesis, indeed, does not take into account the distortions induced by susceptibility artifacts, which depend on the head localization in the magnet. Hence, removing some parts of the brain could penalize them with regard to the remaining ones. Some experiments may also lead to very large activations (and consequently, masks), which would result in more local minima due to the reduction in the number of samples.

Finally, the approach described in this paper could raise some more problems with complex experiments where different brain areas are activated according to different activation profiles. Such experiments call for some improvements of our framework. Anyway, using some robust estimators or mutual information 
could be a simpler choice in such cases. In return, for standard simple clinical brain mapping experiments used in the context of surgery planning, our approach would be very easy to apply and would decrease the risk of false positive disturbing the surgeon thinking.

\section{Conclusion}

In this paper, we have shown that the standard point of view of the neuroimaging community about fMRI alignment should be revisited. A lot of teams, indeed, are in the process of upgrading the field strength of their scanners. The refinements of the registration methods proposed in this paper are relatively simple and should discard any bias related to activations. While a lot of generic robust similarity measures have been proposed for registration, our work has shown that dedicated approaches have to be designed for each problem. Using a priori knowledge about the cognitive experiment, indeed, is the simplest way to get rid of experiment related outliers.

\section{References}

1. Freire, L., and Mangin, J.- F.: Motion correction algorithms may create spurious brain activations in the absence of subject motion. NeuroImage 14, (2001) 709-722

2. Freire, L., Roche, A. and Mangin, J.-F.: What is the best similarity measure for motion correction of fMRI time series?. IEEE Trans. Med. Imag. 21 (2002) 470-484

3. Roche, A.: Recalage d'images médicales par inférence statistique. $\mathrm{PhD}$ Thesis, Université de Nice-Sophia Antipolis, Projet Epidaure, INRIA, (2001)

4. Friston, K. J., Ashburner, J., Frith, C. D., Poline, J.- B., Heather, J. D., and Frackowiak, R. S. J.: Spatial registration and normalization of images. Hum. Brain Mapp. 2 (1995) 165-189

5. Nikou, C., Heitz, F., Armspach, J.-P., Namer, I.- J., and Grucker, D.: Registration of MR/MR and MR/SPECT brain images by fast stochastic optimization of robust voxel similarity measures. NeuroImage 8 (1998) 30-43

6. Woods, R. P., Cherry, S. R., and Mazziotta, J. C.: Rapid automated algorithm for aligning and reslicing PET images. J. Comput. Assist. Tomogr. 16 (1992) 620633

7. Roche, A., Malandain, G., Pennec, X., and Ayache, N.: The correlation ratio as a new similarity measure for multimodal image registration. in Proc. MICCAI'98, 1998, Cambridge, USA, LNCS-1496, Springer Verlag, 1115-1124.

8. Wells, W. M., Viola, P., Atsumi, H., Nakajima, S., and Kikinis, R.: Multi-modal volume registration by maximization of mutual information. Med. Image Anal. 1 (1996) 35-51

9. Maes, F., Collignon, A., Vandermeulen, D., Marchal, G., and Suetens, P.: Multimodality image registration by maximization of mutual information. IEEE Trans. Med. Imag. 16 (1997) 187-198

10. Unser, M., Aldroubi, A., and Eden, M.: B-Spline Signal Processing: Part I-Theory. IEEE Trans. Signal Process. 41 (1993) 821-833

11. Unser, M., Aldroubi, A., and Eden, M.: B-Spline Signal Processing: Part IIEfficient Design and Applications. IEEE Trans. Signal Process. 41 (1993) 834-848 